Three-body forces and shell structure in calcium isotopes

This content has been downloaded from IOPscience. Please scroll down to see the full text. 2012 J. Phys. G: Nucl. Part. Phys. 39085111

(http://iopscience.iop.org/0954-3899/39/8/085111)

View the table of contents for this issue, or go to the journal homepage for more

Download details:

IP Address: 193.190.253.149

This content was downloaded on 06/04/2015 at 08:49

Please note that terms and conditions apply. 


\title{
Three-body forces and shell structure in calcium isotopes
}

\author{
Jason D Holt ${ }^{1,2}$, Takaharu Otsuka ${ }^{3,4}$, Achim Schwenk ${ }^{5,6}$ \\ and Toshio Suzuki ${ }^{7}$ \\ ${ }^{1}$ Department of Physics and Astronomy, University of Tennessee, Knoxville, TN 37996, USA \\ 2 Physics Division, Oak Ridge National Laboratory, PO Box 2008, Oak Ridge, TN 37831, USA \\ ${ }^{3}$ Department of Physics and Center for Nuclear Study, University of Tokyo, Hongo, \\ Tokyo 113-0033, Japan \\ ${ }^{4}$ National Superconducting Cyclotron Laboratory, Michigan State University, East Lansing, \\ MI 48824, USA \\ ${ }^{5}$ ExtreMe Matter Institute EMMI, GSI Helmholtzzentrum für Schwerionenforschung GmbH, \\ 64291 Darmstadt, Germany \\ ${ }^{6}$ Institut für Kernphysik, Technische Universität Darmstadt, 64289 Darmstadt, Germany \\ ${ }^{7}$ Department of Physics, Nihon University, Sakurajosui 3, Tokyo 156-8550, Japan \\ E-mail: jholt31@utk.edu, otsuka@phys.s.u-tokyo.ac.jp, schwenk@physik.tu-darmstadt.de and \\ suzuki@phys.chs.nihon-u.ac.jp
}

Received 13 June 2012

Published 4 July 2012

Online at stacks.iop.org/JPhysG/39/085111

\begin{abstract}
Understanding and predicting the formation of shell structure from nuclear forces is a central challenge for nuclear physics. While the magic numbers $N=2,8,20$ are generally well understood, $N=28$ is the first standard magic number that is not reproduced in microscopic theories with two-nucleon forces. In this paper, we show that three-nucleon forces give rise to repulsive interactions between two valence neutrons that are key to explain ${ }^{48} \mathrm{Ca}$ as a magic nucleus, with a high $2^{+}$excitation energy and a concentrated magnetic dipole transition strength. The repulsive three-nucleon mechanism improves the agreement with experimental binding energies.
\end{abstract}

Communicated by Professor Jacek Dobaczewski

(Some figures may appear in colour only in the online journal)

In nuclei, certain configurations of protons and neutrons (nucleons) are observed to be particularly well bound. These closed-shell or 'magic' nuclei form the basis of the nuclear shell model (exact diagonalizations in spaces based on the observed shell structure [1]), which provides a key computational method in nuclear physics. Exploring the formation of shell structure and how these magic configurations evolve with nucleon number toward the drip lines is a frontier in the physics of nuclei, and a microscopic understanding from nuclear forces presents a major challenge for theory.

The theoretical shortcomings in predicting shell structure are particularly evident in the calcium isotopes. While microscopic calculations with well-established two-nucleon (NN) 
forces reproduce the standard magic numbers $N=2,8,20$, one of the most striking failures is that they do not predict ${ }^{48} \mathrm{Ca}$ as a doubly magic nucleus when neutrons are added to ${ }^{40} \mathrm{Ca}$ $[1,2]$, making $N=28$ the first standard magic number not reproduced in microscopic NN theories. As a result, phenomenological forces have been adjusted to yield a doubly magic ${ }^{48} \mathrm{Ca}[3,4]$, and it has been argued that these phenomenological adjustments may be largely due to neglected three-nucleon ( $3 \mathrm{~N}$ ) forces [5]. Recently, we have shown that $3 \mathrm{~N}$ forces play a decisive role for the oxygen anomaly and can explain why ${ }^{24} \mathrm{O}$ is the heaviest oxygen isotope [6]. In this paper, we present the first study of the impact of $3 \mathrm{~N}$ forces on medium-mass nuclei. Our results demonstrate that one- and two-body contributions from $3 \mathrm{~N}$ forces to valence neutrons, as well as extended valence spaces, are essential to understand the shell structure in the calcium isotopes and $N=28$ as a magic number based on nuclear forces.

Three-nucleon forces were introduced in the pioneering work of Fujita and Miyazawa (FM) [7] and arise because nucleons are finite-mass composite particles that can also be excited by interacting with other particles. The long-range part of $3 \mathrm{~N}$ forces is dominated by the FM $3 \mathrm{~N}$ mechanism, where one nucleon virtually excites a second nucleon to the $\Delta(1232 \mathrm{MeV})$ resonance, which is de-excited by interacting with a third nucleon. Additional long-range and short-range $3 \mathrm{~N}$ interactions are included naturally in chiral effective field theory (EFT) [8], which provides a systematic expansion for nuclear forces. The importance of chiral $3 \mathrm{~N}$ forces has been well established in light nuclei with $A=N+Z \lesssim 12$ [9].

We derive the interactions among valence neutrons following two approaches. First, we use low-momentum interactions $V_{\text {lowk }}$ with smooth cutoffs [10] obtained by evolving a chiral $\mathrm{N}^{3} \mathrm{LO} \mathrm{NN}$ potential [11] to lower resolution with $\Lambda=2.0 \mathrm{fm}^{-1}$. The two-body interactions in the pf and $\mathrm{pfg}_{9 / 2}$ shells are calculated to third order in many-body perturbation theory (MBPT) following [12, 13] in a space of 13 major shells. We use a harmonic oscillator basis with $\hbar \omega=11.48 \mathrm{MeV}$, appropriate for the calcium isotopes. Our results are converged for intermediate-state excitations to $18 \hbar \omega$. Second, we take a standard $G$-matrix that has been used as a starting point in many nuclear-structure calculations [13]. This $G$-matrix is based on the Bonn C NN potential and includes many-body contributions to third order, but with intermediate-state excitations to $2 \hbar \omega$ (and calculated for $\hbar \omega=10 \mathrm{MeV}$ ). Although the $G$-matrix calculation should be improved by extending the intermediate states, this two-body interaction has been used as a standard starting point for shell-model studies, and gives us a baseline to investigate changes due to three-body forces. A detailed study of the cutoff dependence, which provides a measure of the theoretical uncertainty, is left to future work.

To study the validity of the MBPT approach, we have carried out coupled-cluster (CC) calculations for the ground-state energies of ${ }^{40,48,52,54} \mathrm{Ca}$ at the $\Lambda$-CCSD(T) level [14], based on the same $V_{\text {lowk }}$ interaction and basis space. We have verified that the CC energies are converged in these spaces. Using particle-attached (to ${ }^{40} \mathrm{Ca}$ ) $\mathrm{CC}$ energies as single-particle energies (SPEs), the MBPT results agree within a few percent with CC theory (relative to ${ }^{40} \mathrm{Ca}$ ): $-159.3 \mathrm{MeV}(\mathrm{MBPT})$ versus $-155.0 \mathrm{MeV}$ (CC) for ${ }^{48} \mathrm{Ca} ;-230.7 \mathrm{MeV}$ versus $-235.9 \mathrm{MeV}$ for ${ }^{52} \mathrm{Ca}$; and $-259.1 \mathrm{MeV}$ versus $-268.6 \mathrm{MeV}$ for ${ }^{54} \mathrm{Ca}$. This shows that MBPT can be comparable to $\mathrm{CC}$ theory for $V_{\text {lowk }}$ interactions, but also highlights the important role of SPEs.

To understand shell structure in the calcium isotopes, we show in figure 1 the change of the SPEs of the neutron orbitals, with standard quantum numbers $l_{j}$, in the pf and $\mathrm{pfg}_{9 / 2}$ shells as neutrons are added to ${ }^{40} \mathrm{Ca}$. Figure 1 (a) gives the evolution obtained from phenomenological models: GXPF1 [4], a quasi-global fit of two-body matrix elements (starting from a $G$-matrix) and of initial SPEs in ${ }^{41} \mathrm{Ca}$ to experimental data; and KB3G [3], which modifies the monopole part of a standard $G$-matrix. Despite different initial SPEs, as neutrons fill the $\mathrm{f}_{7 / 2}$ shell, the 


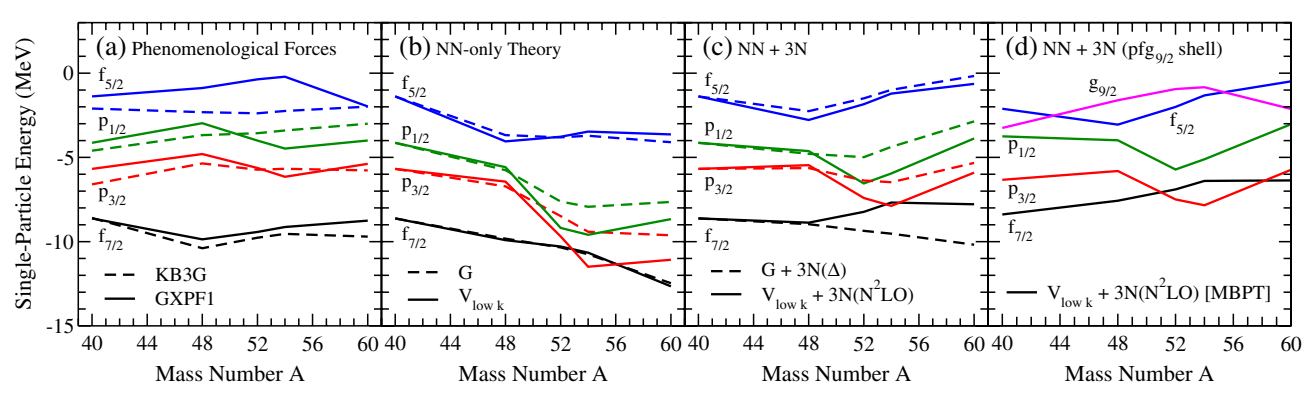

Figure 1. Neutron SPEs relative to the ${ }^{40} \mathrm{Ca}$ energy as a function of mass number $A$. (a) SPEs obtained from phenomenological models KB3G [3] and GXPF1 [4]. (b) NN-only theory: SPEs calculated from a $G$-matrix and low-momentum interactions $V_{\text {lowk }}$. (c) SPEs including contributions from $3 \mathrm{~N}$ forces due to $\Delta$ excitations, $3 \mathrm{~N}(\Delta)$, and chiral EFT $3 \mathrm{~N}$ interactions at $\mathrm{N}^{2} \mathrm{LO}, 3 \mathrm{~N}\left(\mathrm{~N}^{2} \mathrm{LO}\right)$ [20] (see also footnote 8). The results in panels (b) and (c) start from the empirical GXPF1 SPEs in ${ }^{41} \mathrm{Ca}$. (d) SPEs in the $\mathrm{pfg}_{9 / 2}$ shell calculated from $V_{\text {lowk }}$ and $3 \mathrm{~N}\left(\mathrm{~N}^{2} \mathrm{LO}\right)$ forces, where the starting SPEs in ${ }^{41} \mathrm{Ca}$ are calculated consistently in MBPT and include one-body $3 \mathrm{~N}$ contributions.

repulsive interaction between the $\mathrm{f}_{7 / 2}$ and $\mathrm{p}_{3 / 2}$ neutrons causes a significant gap to develop at $N=28$, indicative of a shell closure.

In contrast, both $\mathrm{NN}$-only theories in figure 1(b) exhibit minimal repulsion between these orbitals and the gap remains largely unchanged from ${ }^{40} \mathrm{Ca}$. This is even more evident when the ${ }^{41} \mathrm{Ca}$ SPEs are calculated microscopically at the same third-order level: the NN-only SPEs (based on $V_{\text {lowk }}$ ) are too bound and both $\mathrm{f}_{7 / 2}$ and $\mathrm{p}_{3 / 2}$ orbitals are at $-10.8 \mathrm{MeV}$. Starting from the GXPF1 SPEs in ${ }^{41} \mathrm{Ca}$, the NN-only results in figure 1(b) depend only weakly on the approach or NN forces used, except for differences for $N>28$ in the interactions among the $\mathrm{p}$ orbitals, mostly $\mathrm{p}_{3 / 2}-\mathrm{p}_{3 / 2}$ (due to more attractive second-order core polarization and thirdorder TDA/RPA contributions with $V_{\text {lowk }}, \Lambda=2.0 \mathrm{fm}^{-1}$, compared to the $G$-matrix). This uncertainty remains when $3 \mathrm{~N}$ forces are added (see figure $1(\mathrm{c})$ ). Beyond ${ }^{48} \mathrm{Ca}$, both NNonly theories predict a shell closure at $N=34$, which is a major disagreement between the phenomenological models.

The dominant differences between phenomenological forces and NN-only theory can be traced to their two-body monopole components, which determine the average interaction between orbitals $[2,15]$. In an operator expansion, the monopole interaction corresponds to the term involving number operators, so that differences are enhanced with $N$, and the SPE of orbital $l_{j}$ is effectively shifted by the monopole interaction multiplied by the number of neutrons in orbital $l_{j^{\prime}}^{\prime}$. The interplay of this two-body effect with the initial SPEs largely determines the formation of shell structure.

Next, we include $3 \mathrm{~N}$ forces among two valence neutrons and one nucleon in the core. In [6], we have shown that these configurations give rise to repulsive monopole interactions among excess neutrons. This corresponds to the normal-ordered two-body parts of $3 \mathrm{~N}$ forces, which was found to dominate in CC calculations [16] over residual $3 \mathrm{~N}$ forces (the latter should be weaker because of phase space arguments [17]). For the $G$-matrix approach, we include FM $3 \mathrm{~N}$ forces due to $\Delta$ excitations, $3 \mathrm{~N}(\Delta)$, where the parameters are fixed by standard pion-N- $\Delta$ couplings [18]. For chiral low-momentum interactions, we take into account chiral $3 \mathrm{~N}$ forces at $\mathrm{N}^{2} \mathrm{LO}$ [19]. These include long-range two-pion-exchange parts $c_{i}$ (due to $\Delta$ and other excitations), plus short-range one-pion exchange $c_{D}$ and $3 \mathrm{~N}$ contact $c_{E}$ interactions that have been fit to the ${ }^{3} \mathrm{H}$ binding energy and the ${ }^{4} \mathrm{He}$ matter radius [20] ${ }^{(\text {footnote } 8)}$. For $V_{\text {lowk }}$,

8 We use the $3 \mathrm{~N}$ couplings fit to the ${ }^{3} \mathrm{H}$ binding energy and the ${ }^{4} \mathrm{He}$ radius for the smooth-cutoff $V_{\text {lowk }}$ with $\Lambda=\Lambda_{3 \mathrm{~N}}=2.0 \mathrm{fm}^{-1}$. 

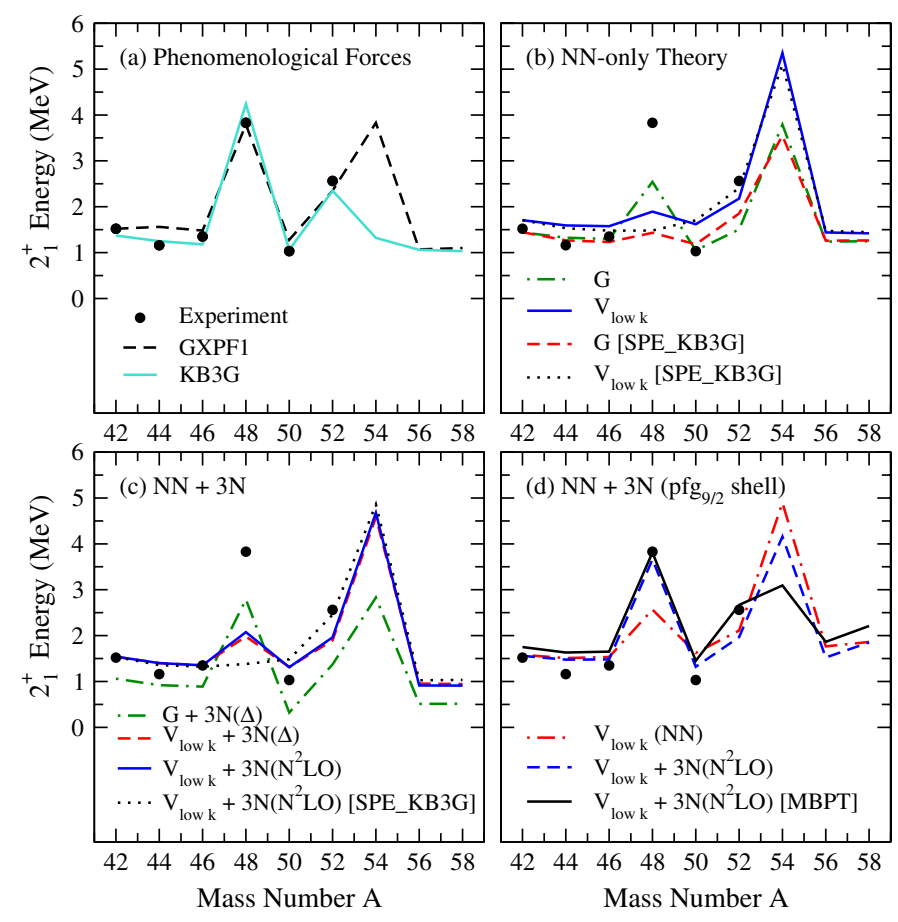

Figure 2. First $2^{+}$excitation energies in the even calcium isotopes compared with experiment. (a) Energies obtained from phenomenological models KB3G [3] and GXPF1 [4]. (b) NN-only theory: energies based on a $G$-matrix and low-momentum interactions $V_{\text {lowk }}$ with empirical GXPF1 SPEs in ${ }^{41} \mathrm{Ca}$, as well as with $\mathrm{KB} 3 \mathrm{G}$ values (SPE_KB3G). (c) Including contributions from $3 \mathrm{~N}$ forces due to $\Delta$ excitations, $3 \mathrm{~N}(\Delta)$ and chiral EFT $3 \mathrm{~N}$ interactions at $\mathrm{N}^{2} \mathrm{LO}, 3 \mathrm{~N}\left(\mathrm{~N}^{2} \mathrm{LO}\right)$ [20] (see also footnote 8). (d) Energies from $V_{\text {lowk }}$ and $3 \mathrm{~N}\left(\mathrm{~N}^{2} \mathrm{LO}\right)$ forces in the $\mathrm{pfg}_{9 / 2}$ shell with empirical GXPF1 SPEs and $\mathrm{g}_{9 / 2}$ at $-1 \mathrm{MeV}$ in ${ }^{41} \mathrm{Ca}$, as well as with SPEs in ${ }^{41} \mathrm{Ca}$ calculated consistently in MBPT.

we also consider the one- $\Delta$ excitation $3 \mathrm{~N}$ force that corresponds to particular values for the two-pion-exchange part $c_{i}$ and $c_{D}=c_{E}=0\left[V_{\text {lowk }}+3 \mathrm{~N}(\Delta)\right]$ [8]. In the $V_{\text {lowk }}+3 \mathrm{~N}$ calculations, we also scale all matrix elements by $\hbar \omega \sim A^{-1 / 3}$.

For all results, full $3 \mathrm{~N}$ multipole contributions are included to first order [21] ${ }^{\text {(footnote 9), }}$, although only the monopole part is responsible for the SPE evolution in figure 1(c). Here we see that in both microscopic approaches, $3 \mathrm{~N}$ forces provide repulsive shifts of all singleparticle levels, changing the binding energies as shown in figure 4 . In addition, the repulsion between the $\mathrm{f}_{7 / 2}$ and $\mathrm{p}_{3 / 2}$ orbitals leads to an increased separation at $N=28$, similar to the phenomenological forces. Moreover, the gap at $N=32$ is increased due to the repulsive $\mathrm{p}_{3 / 2}-\mathrm{p}_{1 / 2}$ interaction, while $N=34$ remains approximately the same.

We take into account many-body correlations by diagonalization in the valence space and plot the first $2^{+}$energy of the even calcium isotopes in figure 2 . The excitation energies of the phenomenological models in figure 2(a) show the fit to the high $2^{+}$energy in ${ }^{48} \mathrm{Ca}$ and hence the doubly magic nature, and highlight the difference in the prediction of $N=34$ as a shell closure. In contrast, ${ }^{48} \mathrm{Ca}$ is not reproduced in any calculation based on $\mathrm{NN}$ forces in figure 2(b), regardless of starting SPEs, or whether we include the $g_{9 / 2}$ orbit in figure 2(d).

9 The first-order $3 \mathrm{~N}$ contribution also dominates the neutron matter energy. 


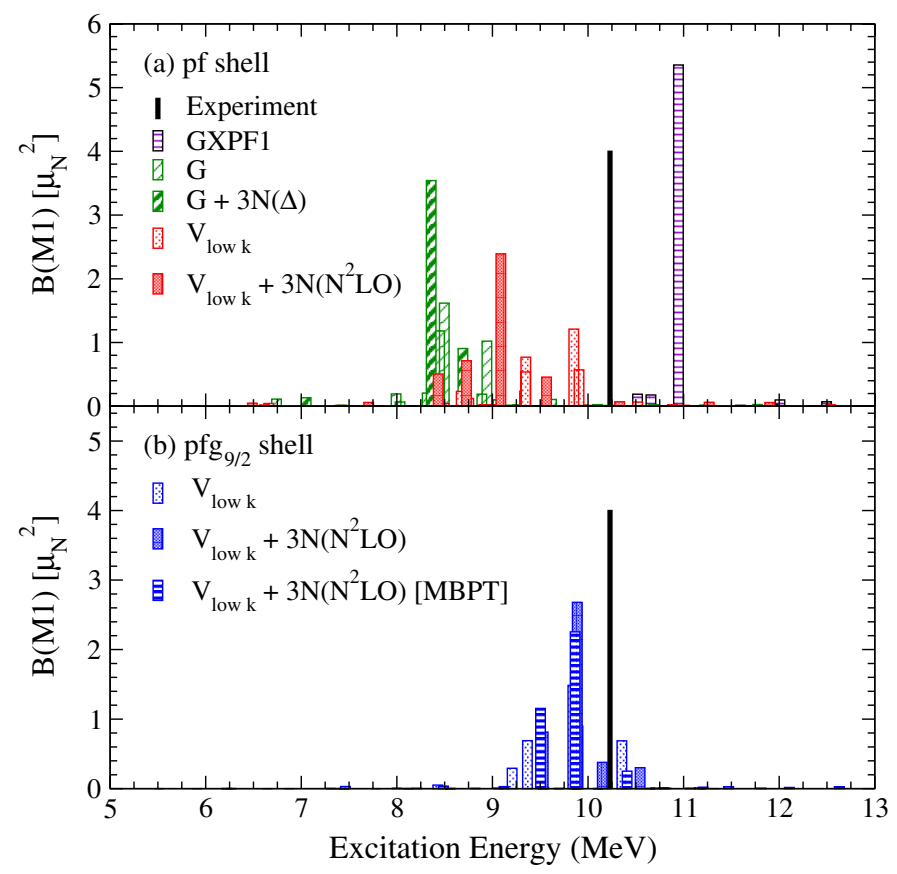

Figure 3. Magnetic dipole transition rates from the ground state to $1^{+}$excited states in ${ }^{48} \mathrm{Ca}$ compared with experiment [24]. The $B(M 1)$ values are calculated in the pf and $\mathrm{pfg}_{9 / 2}$ shells in panels (a) and (b), respectively, based on NN-only interactions and including $3 \mathrm{~N}$ forces (spin $g$ factors are quenched by 0.75 ). The results are labeled as in figure 2 .

With $3 \mathrm{~N}$ forces in figure $2(\mathrm{c})$, the $2^{+}$energy in ${ }^{48} \mathrm{Ca}$ is uniformly improved. The pf shell predictions with $\mathrm{NN}+3 \mathrm{~N}$ forces are similar with initial GXPF1 SPEs, but still below the experimental value. With KB3G SPEs in ${ }^{41} \mathrm{Ca}$, the $2^{+}$energy is significantly lower due to the smaller initial $f_{7 / 2}-p_{3 / 2}$ spacing. When the $g_{9 / 2}$ orbit is included in figure $2(d)$, the $2^{+}$energy is obtained very close to experiment. In addition, we find that all microscopic $\mathrm{NN}$-only and $\mathrm{NN}+3 \mathrm{~N}$ results at this level yield a high $2^{+}$energy in ${ }^{54} \mathrm{Ca}$, and hence a shell closure at $N=34$ (as suggested in [22]). The similarities of $V_{\text {lowk }}+3 \mathrm{~N}(\Delta)$ and $+3 \mathrm{~N}\left(\mathrm{~N}^{2} \mathrm{LO}\right)$ in figure 2(c) demonstrate that the configurations composed of valence neutrons probe mainly the long-range parts of $3 \mathrm{~N}$ forces.

To remove the uncertainty in the initial SPEs, we calculate the SPEs in ${ }^{41} \mathrm{Ca}$ by solving the Dyson equation, consistently including one-body contributions to third order in MBPT in the same space as the two-body interactions, and chiral $3 \mathrm{~N}$ forces between one valence neutron and two core nucleons to first order. In contrast to the failure with NN-only forces, we find in figure 1(d) that the pf shell SPEs are generally similar to the empirical ones, and we find the $g_{9 / 2}$ to initially lie between the $p_{1 / 2}$ and $f_{5 / 2}$ orbitals. Our results based on MBPT SPEs and consistent two-valence-neutron interactions are shown in figure 2(d). The agreement with experiment is very promising for a parameter-free calculation based on $\mathrm{NN}$ and $3 \mathrm{~N}$ forces. Furthermore, the high $2^{+}$in ${ }^{48} \mathrm{Ca}$, despite a relatively small $\mathrm{f}_{7 / 2}-\mathrm{p}_{3 / 2}$ gap, reflects the possible importance of correlations beyond the pf shell (in the context of SPEs, see also [23]). Another challenge for microscopic theories is the prediction of the first excited $\left(1 / 2^{-}\right)$state in ${ }^{49} \mathrm{Ca}$, which indicates the size of the $\mathrm{p}_{3 / 2}-\mathrm{p}_{1 / 2}$ gap at $N=28$. For $V_{\text {lowk }}+3 \mathrm{~N}\left(\mathrm{~N}^{2} \mathrm{LO}\right)$ in both the pf and $\mathrm{pfg}_{9 / 2}$ shells, this energy is $\approx 1.0 \mathrm{MeV}$ compared to the experimental value $2.02 \mathrm{MeV}$, while the MBPT results yield $1.8 \mathrm{MeV}$. 


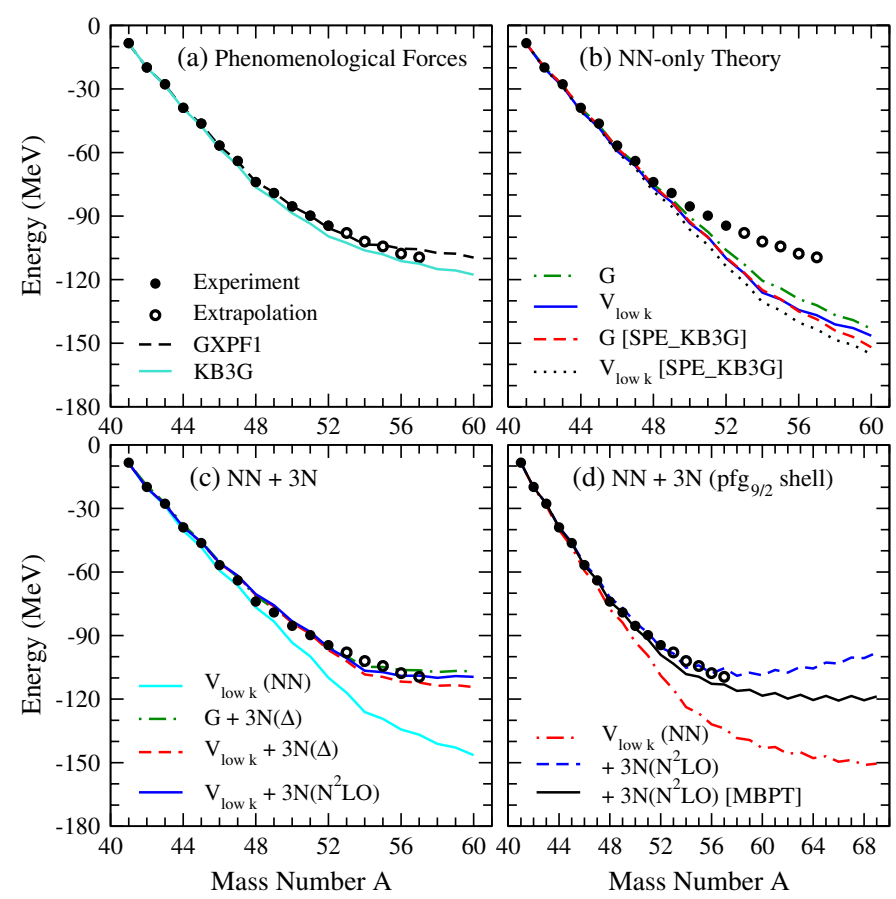

Figure 4. Ground-state energies of calcium isotopes relative to ${ }^{40} \mathrm{Ca}$ compared with experiment and extrapolated energies from the AME2003 atomic mass evaluation [25]. The panels and results are labeled as in figure 2 .

We further examine the closed-shell nature of ${ }^{48} \mathrm{Ca}$ in figure 3, which shows the magnetic dipole transition rates $B(M 1)$ from the $0^{+}$ground state to $1^{+}$excited states, where the experimental concentration of strength indicates a single-particle transition [24]. With NNonly forces, there is a significant fragmentation of strength, and the energy of the dominant transition is below the observed value. When $3 \mathrm{~N}$ forces are included, the peak energies are pushed up for all $3 \mathrm{~N}\left(\mathrm{~N}^{2} \mathrm{LO}\right)$ cases. Moreover, the MBPT results predict a clear concentration, in very good agreement with experiment.

Finally, we turn to the ground-state energies in figure 4, which have been measured to ${ }^{52} \mathrm{Ca}$ [25] and are known to exist to ${ }^{58} \mathrm{Ca}$ [26]. With phenomenological models, the ground-state energies decrease to $N=34$, then the behavior flattens to $N=40$ due to the weakly bound $\mathrm{f}_{5 / 2}$ orbital. With $\mathrm{NN}$-only forces in figure 4 (b) (as expected from figure 1(b)), all neutron-rich calcium isotopes are overbound. In figures $4(\mathrm{c})$ and (d) the repulsion due to $3 \mathrm{~N}$ forces leads to less bound ground-state energies, and all calculations with $3 \mathrm{~N}$ forces exhibit good agreement with experiment (in figure 4 (c) the $V_{\text {low } k}+3 \mathrm{~N}\left(\mathrm{~N}^{2} \mathrm{LO}\right)(\mathrm{KB} 3 \mathrm{G}$ SPE) results would lie on those of $\left.V_{\text {lowk }}+3 \mathrm{~N}(\Delta)(\mathrm{GXPF} 1 \mathrm{SPE})\right)$. The repulsive $3 \mathrm{~N}$ mechanism, discovered for the oxygen anomaly [6], is therefore robust and general for neutron-rich nuclei. In our best calculation with MBPT SPEs in the $\mathrm{pfg}_{9 / 2}$ shell, the ground-state energies are modestly more bound. Our results with $3 \mathrm{~N}\left(\mathrm{~N}^{2} \mathrm{LO}\right)$ suggest a drip line around ${ }^{60} \mathrm{Ca}$, which is close to the experimental frontier [26]. As the predicted energies can significantly flatten from $N=34-40$ as is the case in our best MBPT calculation, the inclusion of continuum effects will be very important.

We have presented the first study of the role of $3 \mathrm{~N}$ forces for binding energies and evolution of shell structure in medium-mass nuclei, thus linking the $3 \mathrm{~N}$ forces frontier to the experimental 
frontier for neutron-rich nuclei. Our results show that $3 \mathrm{~N}$ forces and an extended valence space are key to explain the $N=28$ magic number, leading to a high $2^{+}$excitation energy and a concentrated magnetic dipole transition strength in ${ }^{48} \mathrm{Ca}$. It is intriguing and promising that the parameter-free MBPT results in the extended valence space reproduce experiment best. Future work will include a detailed comparison to empirically adjusted interactions, where the pfg $_{9 / 2}$ interactions can also be transformed into pf-shell-only interactions by an Okubo transformation.

\section{Acknowledgments}

This work was supported by the US DOE grant DE-FC02-07ER41457 (UNEDF SciDAC Collaboration) and DE-FG02-06ER41407 (JUSTIPEN), by grants-in-aid for Scientific Research (A) 20244022 and (C) 22540 290, the JSPS Core-to-Core program EFES, and the Alliance Program of the Helmholtz Association (HA216/EMMI). Part of the numerical calculations have been performed on Kraken at NICS, UT/ORNL, and at the JSC, Jülich.

\section{References}

[1] Caurier E et al 2005 Rev. Mod. Phys. 77427

[2] Poves A and Zuker A 1981 Phys. Rep. 70235

[3] Poves A et al 2001 Nucl. Phys. A 694157

[4] Honma M T et al 2004 Phys. Rev. C 69034335

[5] Zuker A P 2003 Phys. Rev. Lett. 90042502

[6] Otsuka T et al 2010 Phys. Rev. Lett. 105032501

[7] Fujita J and Miyazawa H 1957 Prog. Theor. Phys. 17360

Sakai H et al (ed) 2008 Proc. of the Int. Symp. New Facet of Three Nucleon Force-50 Years of Fujita Miyazawa Three Nucleon Force (FM50) (New York: AIP)

[8] Epelbaum E et al 2009 Rev. Mod. Phys. 811773

[9] Navrátil P et al 2009 J. Phys. G: Nucl. Part. Phys. 36083101

[10] Bogner S K et al 2007 Nucl. Phys. A 78479 Bogner S K et al 2010 Prog. Part. Nucl. Phys. 6594

[11] Entem D R and Machleidt R 2003 Phys. Rev. C 68041001

[12] Kuo T T S and Osnes E 1990 (Lecture Notes in Physics vol 364) (Berlin: Springer) p 1

[13] Hjorth-Jensen M et al 1995 Phys. Rep. 261125

[14] Hagen G et al 2010 Phys. Rev. C 82034330

[15] Otsuka T et al 2010 Phys. Rev. Lett. 104012501

[16] Hagen G et al 2007 Phys. Rev. C 76034302

[17] Friman B and Schwenk A 2011 From Nuclei to Stars: Festschrift in Honor of Gerald E. Brown ed S Lee (Singapore: World Scientific) (arXiv:1101.4858)

[18] Green A M 1976 Rep. Prog. Phys. 391109

[19] van Kolck U 1994 Phys. Rev. C 492932 Epelbaum E et al 2002 Phys. Rev. C 66064001

[20] Bogner S K et al 2009 arXiv:0903.3366

[21] Hebeler K and Schwenk A 2010 Phys. Rev. C 82014314

[22] Otsuka T et al 2001 Phys. Rev. Lett. 87082502

[23] Duguet T and Hagen G 2012 Phys. Rev. C 85034330

[24] von Neumann-Cosel P et al 1998 Phys. Lett. B 4431

[25] Audi G et al 2003 Nucl. Phys. A 729337

[26] Langevin M et al 1983 Phys. Lett. B 130251

Bernas M et al 1997 Phys. Lett. B 415111

Tarasov O B et al 2009 Phys. Rev. C 80034609 